\title{
Nanoemulsions for Food Applications: Development and Characterization
}

\author{
Hélder Daniel Silva • Miguel Ângelo Cerqueira • \\ António A. Vicente
}

Received: 21 May 2011 /Accepted: 1 September 2011 /Published online: 21 September 2011

(C) Springer Science+Business Media, LLC 2011

\begin{abstract}
The application of nanotechnology to food, medical and pharmaceutical industries has received great attention from the scientific community. Driven by the increasing consumers' demand for healthier and safer food products and the need for edible systems able to encapsulate, protect, and release functional compounds, researchers are currently focusing their efforts in nanotechnology to address issues relevant to food and nutrition. Nanoemulsion technology is particularly suited for the fabrication of encapsulating systems for functional compounds as it prevents their degradation and improves their bioavailability. This review focuses on nanoemulsions and provides an overview of the production methods, materials used (solvents, emulsifiers, and functional ingredients) and of the current analytical techniques that can be used for the identification and characterization of nanoemulsions. Finally, nanotechnological applications in foods currently marketed are reported.
\end{abstract}

Keywords Nanoemulsions · Encapsulation · Functional compounds $\cdot$ Analytical methods $\cdot$ Characterization techniques

\footnotetext{
H. D. Silva $\cdot$ M. Â. Cerqueira $\cdot$ A. A. Vicente $(\triangle)$

IBB - Institute for Biotechnology and Bioengineering,

Centre of Biological Engineering, University of Minho,

Campus de Gualtar,

4710-057 Braga, Portugal

e-mail: avicente@deb.uminho.pt

H. D. Silva

e-mail: heldersilva@deb.uminho.pt

M. Â. Cerqueira

e-mail: miguelcerqueira@deb.uminho.pt
}

\section{Introduction}

Nanotechnology is an emerging technology that holds potential to transfigure the food industry (Huang et al. 2010; Luykx et al. 2008). Nanotechnology involves research, technology development, and control of structures within sizes from 1 to $100 \mathrm{~nm}$ (Quintanilla-Carvajal et al. 2010). Materials at the nanometer scale $\left(10^{-9} \mathrm{~m}\right)$ and the development of technology to manipulate or assemble such materials can provide commercial, technological, and scientific opportunities for the industry (Huang et al. 2010). The application of nanotechnology to the food field may allow the modification of many macroscale characteristics of food, such as texture, taste, other sensory attributes, coloring strength, processability, and stability during shelf life, leading to a great number of new products. Nanotechnology can also improve the water solubility, thermal stability, and oral bioavailability of functional compounds (Huang et al. 2010; McClements et al. 2009, 2007).

Functional compounds, claimed to provide health benefits as prevention or treatment of diseases (Chu et al. 2007), are already available in the marketplace in the form of capsules or tablets. Nevertheless, it has become evident that these solutions may not sustain the health benefits of these functional compounds, mainly because of their low bioavailability. This is particularly the case of lipophilic compounds (Chen et al. 2006; Chu et al. 2007; Spernath and Aserin 2006). Improving the bioavailability of functional compounds often means enhancing the absorption in the gastro-intestinal tract, this being a critical requirement. The progress in nanotechnology offers possible solutions for improving water solubility and bioavailability of, e.g., lipophilic functional compounds (Chu et al. 2007).

Nanoemulsions are one of the most interesting fields of application, once they can act as carriers or delivery systems 
for lipophilic compounds, such as nutraceuticals, drugs, flavors, antioxidants, and antimicrobial agents (Jiahui et al. 2004; Kesisoglou et al. 2007; McClements et al. 2007; Sanguansri and Augustin 2006; Weiss et al. 2008; Wissing et al. 2004). Nanoemulsions consist of a lipid phase dispersed in an aqueous continuous phase, with each oil droplet being surrounded by a thin interfacial layer consisting of emulsifier molecules (Acosta 2009; McClements et al. 2007; Tadros et al. 2004). Usually, nanoemulsions are highly stable to gravitational separation because the relatively small particle size means that Brownian motion effects dominate gravitational forces (McClements 2005). They also have good stability against droplet aggregation because the range of attractive forces acting between the droplets decreases with decreasing particle size, while the range of steric repulsion is less dependent on particle size (McClements 2005; Tadros et al. 2004). Nanoemulsion-based delivery systems can also improve the bioavailability of the encapsulated components due the small particle size and high surface-to-volume ratio (Acosta 2009). Various methods have been developed to prepare lipophilic functional compounds with particle diameters in the nano-size range. Such methods include emulsification-evaporation, emulsification-diffusion, solvent displacement, and precipitation, and can be classified as high- or low energy approaches (Acosta 2009; Chu et al. 2007; Leong et al. 2009; Tadros et al. 2004). As the name suggests, high-energy methods make use of devices that use very high mechanical energy; these systems offer more control of the size distribution and composition of the resultant nanoemulsions, but some chemicals can be easily degraded during the production process; further, most production processes are not prepared to be scaled up. On the other side is low-energy emulsification; this technique requires low energy for nanoemulsions production and mainly depends on the intrinsic physicochemical properties of surfactants and the oily phase, and its scale-up is more straightforward (Date et al. 2010).

Although most of the methods are in the development stage, they enable preparing extremely fine particulate nanodispersions, and allow continuous and controllable production (Chu et al. 2007; Horn and Rieger 2001). In order to stabilize the nanoemulsions an emulsifier or a combination of emulsifiers is needed. The emulsifier molecules adsorb at the interface between the two phases, lowering the interfacial tension and preventing or slowing down the aggregation of particles of the dispersed phase by increasing repulsion forces between them. The emulsifiers range from proteins to cationic, anionic or non-ionic surfactants, and even polysaccharides (Chu et al. 2007).

This review revisits nanoemulsion production methods (high- and low energy) as well as the materials used for nanoemulsion preparation (solvents and emulsifiers, as well as functional compounds) that are already in use or can be used.
A description of analytical techniques used for nanoemulsions characterization is also provided; these techniques were divided in three categories: separation techniques (such as high-pressure liquid chromatography-HPLC and flow field fractionation-FFF), physical techniques (such as dynamic light scattering-DLS, zeta potential, differential scanning calorimetry-DSC, Fourier transform infrared-FTIR, nuclear magnetic resonance-NMR and X-ray diffraction-XRD) and imaging techniques (such as transmission electron microscopy-TEM, scanning electron microscopy-SEM and atomic force microscopy-AFM). Despite some of these techniques being mostly applied to nanoparticles, they can be applied to characterize nanoemulsions as well.

Finally, examples of nanotechnology-based foods and food ingredients are presented which are being marketed or very close from it, aiming at demonstrating actual applications and opening the door for further uses of this type of products by the food industry.

\section{Production of Nanoemulsions}

When one of two immiscible liquid phases is dispersed as droplets, the resulting mixture is referred to as an emulsion (Akoh and Min 2002). Nanoemulsions consist of oil droplets in the nano-ranged size, between 10 and $100 \mathrm{~nm}$ dispersed within an aqueous continuous phase, with each oil droplet surrounded by surfactant molecules (Acosta 2009; McClements et al. 2009, 2007).

Nanoemulsions can be produced using a variety of methods, which are classified as either high-energy or lowenergy approaches (Acosta 2009; Leong et al. 2009; Tadros et al. 2004). High-energy approaches use mechanical devices capable of generating intense disruptive forces that breakup the oil and water phases and lead to the formation of oil droplets, e.g., high-pressure valve homogenizers, microfluidizers, and sonication methods (Gutiérrez et al. 2008; Leong et al. 2009; Velikov and Pelan 2008; Wooster et al. 2008). Low-energy approaches rely on the spontaneous formation of oil droplets within mixed oil-water-emulsifier systems when the solution or environmental conditions are altered, e.g., phase inversion and solvent demixing methods (Anton et al. 2008; Bouchemal et al. 2004; Chu et al. 2007; Freitas et al. 2005; Tadros et al. 2004; Yin et al. 2009). Table 1 presents nano-emulsification techniques reported in the literature. In the following sections high-energy and lowenergy approaches will be detailed.

\section{High-energy Approaches}

The formation of nanoemulsions by high-energy methods is governed by the selected composition (i.e., surfactants and functional compounds) and by the quantity of energy 


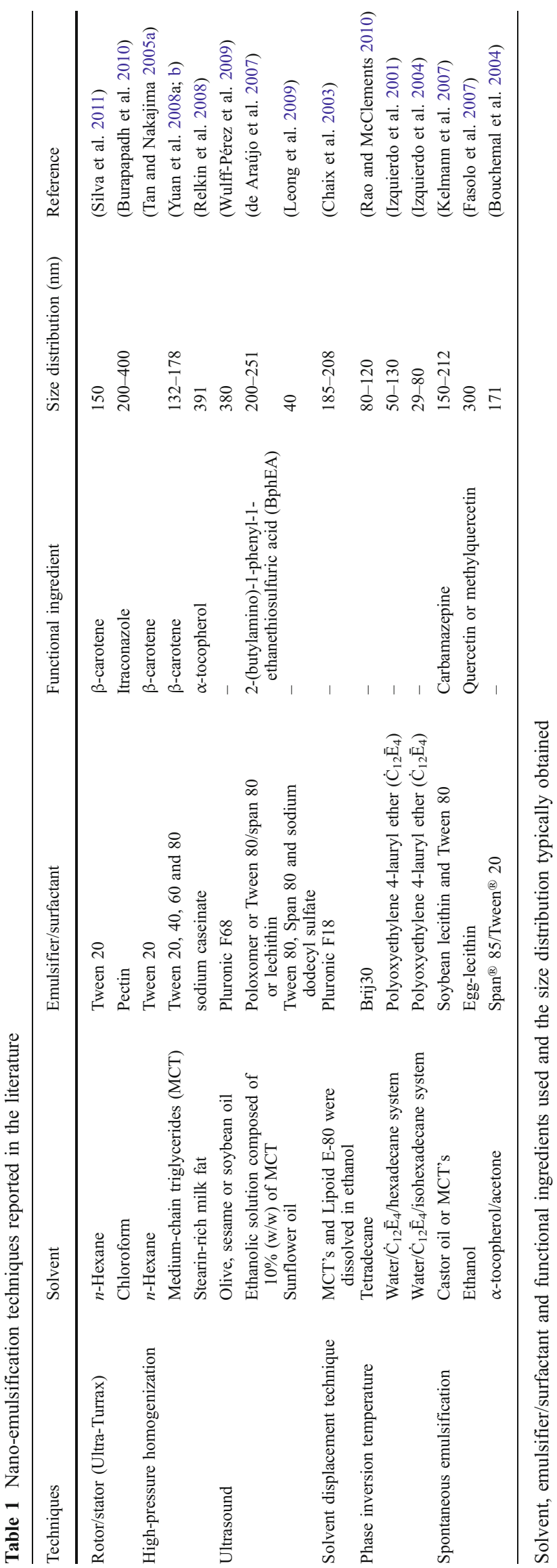

applied. Therefore, nanoemulsions produced through highenergy methods present a natural predisposition to preserve the nanoemulsions formation against formulation modification-e.g., addition of monomer, surfactant, co-surfactant, etc. (Anton et al. 2008).

The mechanical processes generating nanoemulsions can be divided into three major groups based on the used devices (Anton et al. 2008; Sanguansri and Augustin 2006) that are presented below:

- High-pressure homogenization - in high-pressure homogenization, the mixture is subject to very high pressures and is pumped through a restrictive valve. The very high shear stress causes the formation of very fine emulsion droplets (Quintanilla-Carvajal et al. 2010; Sanguansri and Augustin 2006);

- Ultrasound - when two immiscible liquids are submitted to high-frequency sound waves in the presence of a surfactant, emulsion droplets are formed by cavitation. This causes intense shock waves in the surrounding liquid and the formation of liquid jets at high speed is responsible for the formation of emulsion droplets. However, this technology has not yet been proved as efficient for industrial-scale applications (Maa and Hsu 1999; Quintanilla-Carvajal et al. 2010; Sanguansri and Augustin 2006).

- High-speed devices-rotor/stator devices (such as Ultra-Turrax) when compared with the other highenergy approaches do not provide a good dispersion in terms of droplet sizes. With the energy provided mostly being dissipated, generating heat (Anton et al. 2008; Walstra 1993).

Regarding the formation of nanoemulsions by highenergy approaches, most of the energy provided is dissipated in the form of heat. Though this idea is generalized, only very few authors report energy calculations in their publications. Tadros et al. (2004), present energy calculations for a high-pressure homogenization. They reported an efficiency of $0.1 \%$ (where $99.9 \%$ is dissipated as heat) during the homogenization process for the production of an emulsion with $600 \mathrm{~nm}$ of diameter using a surfactant with $10 \mathrm{mN} \mathrm{m}^{-1}$ of interfacial tension (Tadros et al. 2004). It is noteworthy to mention that scaling-up these values to industrial-scale applications may be problematic and lead to significant errors. Determinations at pilot-scale are, therefore, highly recommended.

\section{Low-energy Approaches}

In low-energy approaches, nanoemulsions are obtained as a result of phase transitions produced during the emulsification process which is carried out, generally, at constant temperature and changing the composition (Usón et al. 2004) or at constant composition and changing the 
temperature (Izquierdo et al. 2001; Morales et al. 2003). The methods used more often are presented below:

- Membrane emulsification -it is a low-energy process that requires less surfactant (when compared with highenergy methods) and produces emulsions with a narrow size distribution range. This method involves formation of a dispersed phase (droplets) through a membrane into a continuous phase. Nevertheless, this method has as limitation the low flux of the dispersed phase through the membrane, this being an issue during scale-up (Sanguansri and Augustin 2006);

- Spontaneous emulsification-this mechanism occurs when an organic phase and an aqueous phase are mixed, with the organic phase being a homogeneous solution of oil, lipophilic surfactant and water-miscible solvent, and the aqueous phase consisting of water and hydrophilic surfactant (Bouchemal et al. 2004). The spontaneous features of this method result of the initial non-equilibrium states of two bulks liquids when they are brought into contact without stirring. It is only under specific conditions that spontaneous emulsification occurs. Spontaneous emulsification is produced by different mechanisms (e.g., diffusion of solutes between two phases, interfacial turbulence, surface tension gradient, dispersion mechanism, condensation mechanism) which seem to be affected by the systems' compositions and their physicochemical characteristics like the physical properties of the oily phase and nature of the surfactants (Bouchemal et al. 2004). This process itself increases entropy and thus decreases the Gibbs free energy of the system (Anton et al. 2008);

- Solvent displacement - this method consists of mixing a water-miscible organic solvent containing lipophilic functional compounds in an aqueous phase containing an emulsifier. The rapid diffusion of the organic solvent in the aqueous phase promotes the formation of nanoemulsions enabling their preparation in one step at low-energy input with high yield of encapsulation. Finally, the organic solvent is removed from the nanodispersion under reduced pressure. Nevertheless the use of this technique is limited to water-miscible solvents (Chu et al. 2007; Yin et al. 2009);

- Emulsion inversion point - this method consists in varying the composition of the system at a constant temperature. The structures are formed through a progressive dilution with water or oil in order to create kinetically stable nanoemulsions (Anton et al. 2008; Sadtler et al. 2010);

- Phase inversion point - this method uses the specific ability of surfactants (non-ionic) to alter their affinities to water and oil in function of temperature at a fixed composition (Shinoda and Saito 1968, 1969). It consists in suddenly breaking-up the microemulsions maintained at the phase inversion point by a rapid cooling
(Izquierdo et al. 2001; Izquierdo et al. 2004; Sadurní et al. 2005) or by a dilution in water or oil (Anton et al. 2008, 2007). The nanoemulsions immediately formed are kinetically stable and can be considered as irreversible. This process is relatively simple, prevents the encapsulated drug being degraded during processing, consumes low amounts of energy and allows an easy industrial scale-up (Anton et al. 2008).

\section{Materials used in Nanoemulsions Production}

Solvents

Many of the techniques listed above are co-dependent of solvents, in a way that these solvents are fundamental for nanoemulsions production. Table 2 shows the most used solvents, some of which are not food grade or generally recognized as safe or, if they are, they are not well accepted by the consumers, this being an issue that needs to be improved. Solvents like $n$-hexane and others may therefore be replaced by, e.g., sunflower oil, MCTs, etc.

\section{Emulsifiers/Surfactants}

Various physical factors can affect or disturb the stability of emulsion droplets and affect their features as carrier of functional ingredients. Therefore, stabilization of emulsions is very important in order to provide a better stability (either kinetic or thermodynamic) over conventional emulsions, a more efficient encapsulation of functional compounds and a better bioavailability (Grigoriev and Miller 2009; Huang et al. 2010).

The most common way to stabilize emulsions is by surfactant adsorption at the interface between droplets and the dispersion medium, reducing the interfacial tension. Depending of the class of surfactant applied, different ways of promoting stability can be achieved. Ionic surfactants can provide an electrical charge to the emulsion droplets and non-ionic surfactants, creating a steric barrier via bulky molecular groups directed towards the dispersion medium (Grigoriev and Miller 2009). Table 3 shows the emulsifiers/ surfactants more frequently used, their type and charge. Non-ionic surfactants are the most used in research studies.

Functional Compounds

Nanoemulsions can encapsulate a diverse range of lipophilic compounds, including bioactive lipids, flavors, antimicrobials, antioxidants, and drugs (Chen et al. 2006; McClements et al. 2007; Shefer and Shefer 2003; Ubbink and Krüger 2006) leading to the increase of their bioactivity, desirability, and palatability (McClements et al. 2007). 
Table 2 Solvents used in nanoemulsions production, corresponding functional ingredient and classification

\begin{tabular}{|c|c|c|c|c|}
\hline Solvents & $\begin{array}{l}\text { Presence allowed } \\
\text { in foods }\end{array}$ & $\begin{array}{l}\text { Maximum values } \\
\text { allowed (ppm) }\end{array}$ & $\begin{array}{l}\text { Functional } \\
\text { Compounds }\end{array}$ & References \\
\hline Hexadecane & No & - & - & (Izquierdo et al. 2001) \\
\hline$n$-Decane & No & - & - & (Ee et al. 2008) \\
\hline Isohexadecane & No & - & - & (Izquierdo et al. 2004) \\
\hline Chloroform & No & - & Itraconazole & (Burapapadh et al. 2010) \\
\hline$n$-Hexane & Yes & 50 & $\beta$-carotene & $\begin{array}{l}\text { (Tan and Nakajima 2005a; The Commission } \\
\text { Of The Eureopean Communities 1995) }\end{array}$ \\
\hline Tetradecane & Yes & 50 & - & $\begin{array}{l}\text { (Rao and McClements 2010; } \\
\text { The good scents company, 2011) }\end{array}$ \\
\hline Ethyl acetate & Yes & 50 & - & $\begin{array}{l}\text { (Lee and McClements 2010; The Commission } \\
\text { Of The Eureopean Communities 1995) }\end{array}$ \\
\hline Acetone & Yes & 50 & $\beta$-carotene & $\begin{array}{l}\text { (The Commission Of The Eureopean } \\
\text { Communities 1995; Yin et al. 2009) }\end{array}$ \\
\hline $\mathrm{MCT}$ & Yes & & $\beta$-carotene & (Yuan et al., 2008a; Yuan et al., 2008b) \\
\hline Sunflower oil & Yes & & - & (Leong et al. 2009) \\
\hline Corn oil & Yes & & - & (Lee and McClements 2010) \\
\hline Ethanol & Yes & & $\beta$-carotene & (Pan et al. 2007) \\
\hline Octanoic acid & Yes & & - & (Katagi et al. 2007) \\
\hline Stearin-rich milk fat & Yes & & $\alpha$-tocopherol & (Relkin et al. 2008) \\
\hline Olive oil & Yes & & - & (Wulff-Pérez et al. 2009) \\
\hline Soybean oil & Yes & & - & (Wulff-Pérez et al. 2009) \\
\hline Sesame oil & Yes & & - & (Wulff-Pérez et al. 2009) \\
\hline Paraffin oil & Yes & & - & (Liu et al. 2006) \\
\hline D-Limonene & Yes & & - & (Jafari et al. 2007) \\
\hline Caprylic acid & Yes & & - & (Katagi et al. 2007) \\
\hline
\end{tabular}

The major lipophilic functional compounds that more often need to be incorporated in foods can be divided into four categories: fatty acids (e.g., omega three fatty acids), carotenoids (e.g., $\beta$-carotene), antioxidants (e.g., tocopherol) and phytosterols (e.g., stigmasterol). Table 4 shows a list of lipophilic functional compounds that have been encapsulated into nanoemulsion systems, their expected benefits and their fields of application.

\section{Techniques for the Identification and Characterization of Nanoemulsions}

Detection, identification, and characterization of nanodelivery systems are essential for the understanding of the benefits as well as the potential toxicity of these systems (Luykx et al. 2008). In this section, the analytical techniques that can be used for the identification and characterization of nanoemulsions are described. The analytical approaches have been subdivided into three groups: separation, characterization, and imaging techniques. The principles of the techniques together with their advantages and drawbacks are reported.

\section{Separation Techniques}

Some analytical techniques can be used for in situ identification of nanoemulsions; nevertheless, in most cases it is not possible to detect them in the food matrixes. Therefore, separation techniques are necessary to isolate the nanoemulsions from food prior to their characterization. This section describes the most important separation techniques for isolation of nanoemulsions.

\section{Chromatography}

Because size and/or charge are typical characteristics of nanoemulsions, size-exclusion chromatography (SEC) and/ or ion exchange chromatography (IEC) are the most suitable types of liquid chromatography for the separation of nanoemulsions from the food matrix. In the case of SEC, compounds are separated on the basis of size; larger molecules elute faster than smaller ones. However, elution could be affected by the shape of the compound. In the case of IEC, compounds are separated on the basis of charge; low-charged compounds elute faster than highly charged ones (Luykx et al. 2005, 2008). 
Table 3 Emulsifiers/surfactants used in nanoemulsions production and respective type

\begin{tabular}{|c|c|c|}
\hline Emulsifiers/surfactants & Type & References \\
\hline Polyethylene glycol (35) castor oil (Cremophor EL ${ }^{\mathbb{R}}$ ) & Surfactant (non-ionic) & (Usón et al. 2004) \\
\hline Polyoxyehtylene-660-12-hydroxy stearate & Surfactant (non-ionic) & (Anton et al. 2007) \\
\hline Polyoxyethylene 4-lauryl ether & Surfactant (non-ionic) & (Izquierdo et al. 2004) \\
\hline Span 20 & Surfactant (non-ionic) & (Porras et al. 2008) \\
\hline Span 80 & Surfactant (non-ionic) & (Porras et al. 2008) \\
\hline Tween 20 & Surfactant (non-ionic) & (Porras et al. 2008) \\
\hline Tween 80 & Surfactant (non-ionic) & (Porras et al. 2008) \\
\hline Tween 40 & Surfactant (non-ionic) & (Yuan et al., 2008b) \\
\hline Tween 60 & Surfactant (non-ionic) & (Yuan et al., 2008b) \\
\hline Sucrose fatty acid ester (L1695) & Surfactant (non-ionic) & (Yin et al. 2009) \\
\hline Polyoxyethylene 6-lauryl ether & Surfactant (non-ionic) & (Izquierdo et al. 2005) \\
\hline Decaglycerol monolaurate (ML750) & Surfactant (non-ionic) & (Yin et al. 2009) \\
\hline Polyglycerol esters of fatty acids (PGEs) & Surfactant (non-ionic) & (Tan and Nakajima 2005b) \\
\hline Alkanol-XC & Surfactant (anionic) & (Howe and Pitt 2008) \\
\hline Sodium dodecyl sulfate & Surfactant (anionic) & (McClements 2000) \\
\hline Dodecyl trimethylammonium bromide & Surfactant (cationic) & (McClements 2000) \\
\hline Gelatin & Protein (amphiphilic) & (Ribeiro et al. 2008) \\
\hline Whey protein isolate (WPI) & Protein (amphiphilic) & (Lee and McClements 2010) \\
\hline Whey protein concentrate (WPC) & Protein (amphiphilic) & (Chu et al. 2007) \\
\hline Whey protein hydrolysate (WPH) & Protein (amphiphilic) & (Chu et al. 2007) \\
\hline Sodium caseinate $(\mathrm{SC})$ & Protein (amphiphilic) & (Yin et al. 2009) \\
\hline Modified starch & Hydrocolloid (cationic) & (Jafari et al. 2007) \\
\hline Pectin & Hydrocolloid (anionic) & (Burapapadh et al. 2010) \\
\hline Lipoid S75-3 ${ }^{\circledR}$ & Phospholipid (amphiphilic) & (Anton et al. 2007) \\
\hline
\end{tabular}

HPLC is a form of liquid chromatography to separate, analyse, and quantify compounds that are dissolved in solution. Compounds are separated by injecting the sample mixture (carried by mobile phase) onto a column. Depending on the type of stationary phase, compounds can be separated based on their charge (weak/strong cation or anion exchange chromatography), molecular mass (size-exclusion chromatography), hydrophobicity/polarity (reversed-phase HPLC, hydrophobic interaction chromatography), and specific characteristics (affinity chromatography). The most common detectors for HPLC are ultraviolet-visible (UV-Vis) light absorbance, fluorescence, electrochemical, and diffraction detectors (Luykx et al. 2008). HPLC has been used in food analysis for measuring numerous compounds, for example, carbohydrates, vitamins, additives, mycotoxins, amino acids, proteins, tryglycerides in fats and oils, lipids, chiral compounds, and pigments. It is a straightforward, robust, and reproducible technique. Sensitive selective detectors are available for HPLC, and their selection depends mostly on the compound to be analyzed (Luykx et al. 2008). HPLC can also be used to quantify functional compounds encapsulated in the nanoemulsions: $\beta$-carotene (Tan and Nakajima 2005a, b); (Yin et al. 2009), $\alpha$-tocopherol (Cheong et al. 2008) and thalidomide (Araújo et al. 2011).

\section{Field Flow Fractionation}

FFF is a flow-assisted technique for the separation of analytes, from macromolecules, such as proteins (nanometer range), to micrometer-sized particles, such as whole cells. In a one single run, very broad ranges of molecular sizes can be separated (Luykx et al. 2008). FFF can also separate particles due to their Stokes radius (Dulog and Schauer 1996; Jores et al. 2004). In this technique, smaller particles are transported faster and elute earlier if a parabolic flow is used; if a cross flow is used with particles of the same volume and different shape, the isometric particles will be eluted first than the asymmetric particles (Jores et al. 2004). FFF has advantages over other separation techniques such as higher biocompatibility, reduction of sample carry-over, and simple sterility issues. The separation times typically range from a few minutes up to $30 \mathrm{~min}$. Despite these advantages, the FFF has the disadvantage of being easily overloaded by higher concentrations of the compounds. In order to overcome this, dilutions should be made. Nevertheless, the detection of trace amounts of compounds is limited by the sensitivity of an appropriate detector system (Luykx et al. 2008). 
Table 4 Examples of functional compounds encapsulated into nanoemulsion systems, their benefits and principal applications

\begin{tabular}{|c|c|c|c|}
\hline Functional compounds & Benefits & Application & References \\
\hline$\alpha$-Tocopherol & Antioxidant & Medicine & (Cheong et al. 2008) \\
\hline$\beta$-carotene & Antioxidant, vitamin A precursor & Natural food color & (Yin et al. 2009) \\
\hline Curcumin & $\begin{array}{l}\text { Antioxidant, anti-inflammatory and } \\
\text { anticarcinogenic }\end{array}$ & Natural food color & (Huang et al. 2010) \\
\hline Phytosterols & Cholesterol absorption inhibitor & $\begin{array}{l}\text { Medicine, cosmetic and } \\
\text { as food additive }\end{array}$ & (Garti et al. 2005) \\
\hline Lycopene & $\begin{array}{l}\text { Antioxidant, potential agent for prevention } \\
\text { of some types of cancers, particularly } \\
\text { prostate cancer }\end{array}$ & Natural food color & (Garti et al. 2005) \\
\hline Lidocaine & Local anesthetic and antiarrhythmic drug & Medicine & (Sadurní et al. 2005) \\
\hline Itraconazole & Antifungal agent & Medicine & (Burapapadh et al. 2010) \\
\hline $\begin{array}{l}\text { 2-(butylamino)-1-phenyl-1- } \\
\text { ethanethiosulfuric acid (BphEA) }\end{array}$ & Schistosomicidal drug & Medicine & (de Araújo et al. 2007) \\
\hline Carbamazepine & Anticonvulsant and mood stabilizing drug & Medicine & (Kelmann et al. 2007) \\
\hline Quercetin & Anti-inflammatory and antioxidant properties & Medicine & (Fasolo et al. 2007) \\
\hline Cisplatin & Anti-cancer drug & Medicine & (Velinova et al. 2004) \\
\hline Thalidomide & Immunomodulatory agent & Medicine & (Araújo et al. 2011) \\
\hline
\end{tabular}

\section{Physical Characterization Techniques}

This section describes the techniques that are used to characterize nanoemulsions from a physical perspective (e.g., size, size distribution, zeta potential, and crystallinity of the nanoemulsions).

\section{Dynamic Light Scattering}

DLS also known as photon correlation spectroscopy or quasi-elastic light scattering is a technique used for rapid determination of the size distribution profile of small particles in suspensions or polymers in solution. DLS measures Brownian motion and relates this to the size of the particles through Stokes-Einstein equation. Through the illumination of the particles with a laser and analyzing the intensity fluctuations in the scattered light, DLS allows calculating the size of the particles. DLS provides a fast and adequate evaluation of the size of nanoemulsions and is often used to evaluate the size distribution of nanoemulsions, as well their size stability through storage (Araújo et al. 2011; Preetz et al. 2010; Silva et al. 2011).

\section{Zeta Potential}

Zeta potential is a scientific term for electrokinetic potential in colloidal systems (Mills et al. 1993). In colloidal chemistry literature, zeta potential is the potential difference between the dispersion medium and the stationary layer of fluid attached to the dispersed particle. A value of $30 \mathrm{mV}$ (positive or negative) can be taken as the arbitrary value that separates low-charged surfaces from highly charged surfaces (Preetz et al. 2010). Zeta potential value can be related to the stability of colloidal dispersions, indicating the degree of repulsion between adjacent, similarly charged particles in dispersion. For molecules and particles that are small enough, a high zeta potential will confer stability, i.e., the solution or dispersion will resist to aggregation. When the potential is low, attraction exceeds repulsion and the dispersion will break and flocculate. So, colloids with high zeta potential (negative or positive) are electrically stabilized while colloids with low zeta potentials tend to coagulate or flocculate. Briefly, zeta potentials from 0 to $\pm 30 \mathrm{mV}$ indicate instability, while zeta potentials higher than $\pm 30 \mathrm{mV}$ indicate stability (ASTM 1985). The zeta potential of nano-scaled particles is influenced by many factors, such as the source of particles and the treatment with different surfactants, electrolyte concentration (ionic strength), particle morphology and size, $\mathrm{pH}$ of the solution and state of hydration (Simunkova et al. 2009). For instance, Araújo et al. (2011), Gao et al. (2011), and Preetz et al. (2010), evaluated the zeta potential of nanoemulsions: the information given by the zeta potential allows stating that the nanoemulsions with highly charged surfaces are stable and will resist to droplet aggregation.

\section{Differential Scanning Calorimetry}

DSC is a thermo-analytical technique in which the difference in the amount of heat required to increase the temperature of a sample and reference is measured as a function of temperature. Both the sample and reference are maintained at nearly the same temperature throughout the experiment. Generally, the temperature program for a DSC analysis is designed such that the sample holder temperature increases linearly as a function of time. The reference sample should have a well-defined heat capacity over the 
range of temperatures to be scanned (Salmah et al. 2008; Venturini et al. 2011). DSC can be used to detect phase transitions including the melting of crystalline regions, and to analyse the proportion of solid fat or the proportion of ice crystals in emulsions (Thanasukarn et al. 2004). Thanasukarn et al. (2004) shows that fat crystallization affects the emulsion stability depending on the emulsifier used. They showed that the thermal decomposition follows the melting of the drug encapsulated (Thanasukarn et al. 2004). Usón et al. (2004) have shown that DSC can be used to determine the crystallization temperature of a mixture of surfactants.

\section{Fourier Transform Infrared}

FTIR spectroscopy is based in an infrared radiation that passes through a sample where it is mostly absorbed by the sample and some of it is transmitted. The resulting spectrum represents the molecular absorption and transmission, creating a molecular fingerprint of the sample. Each sample fingerprint presents its characteristic absorption peaks that correspond to the frequencies of vibrations between the bonds of the atoms of the material. Because each different material is a unique combination of atoms, no two compounds produce the exact same infrared spectrum (Nicolet 2001). Therefore, infrared spectroscopy can result in a positive identification of different materials. In addition, the size of the peaks in the spectrum is a direct indication of the amount of material present in the sample. The major advantages of FTIR are the fact that it can determine the amount of components in a mixture, it can determine the quality or consistency of a sample, the small time required for analyses (because all frequencies are measured simultaneously), the fact that it is a very sensitive method, relatively simple to work with and internally calibrated. These advantages make measurements made by FTIR extremely accurate and reproducible (Nicolet 2001). Araújo et al. (2011) report the encapsulation of thalidomide in nanoemulsions by spontaneous emulsification, where the crystallization process was studied through FTIR analysis of the crystals; when present in a nanoemulsion, these crystals were found to be in a different polymorphic form than that found before nanoemulsions preparation.

\section{Nuclear Magnetic Resonance}

NMR is a powerful and complex analytical tool that allows the study of compounds in either liquid or solid state and serves equally in quantitative as in structural analysis. It is very efficient in gathering structural information regarding molecular compounds. It can be used as a complementary technique to methods of optical spectroscopy and mass spectrometry leading to precise information concerning the structural formula, stereochemistry, information about the preferred conformation of molecules; it may also be used to identify the compound in study (Rouessac and Rouessac 2007). The application of NMR to nanoemulsions characterization has been only slightly exploited. Jenning et al. (2000) successfully incorporated medium-chain triglycerides oil in a matrix of a solid long-chain glyceride (glyceryl behenate) and the structure of the liquid lipids inside the matrix of solid lipid nanoparticles was characterized through ${ }^{1} \mathrm{H}$ NMR. Information about the mobility, the arrangement and the environment of the oil molecules (Jenning et al. 2000b) was obtained. Casadei et al. (2006), determined the amount of unloaded ibuprofen and the entrapment efficiency of this compound within solid lipid particles through ${ }^{1} \mathrm{H}$ NMR analysis.

\section{X-Ray Diffraction}

XRD techniques are a family of non-destructive analytical techniques that reveals information about the crystallographic structure, chemical composition and physical properties of materials. XRD is based on observing the scattered intensity of an X-ray beam hitting a sample as a function of incident and scattered angles, polarization and wavelength or energy (Azároff et al. 1974). XRD is mostly used for the identification of crystalline compounds by their diffraction pattern. Nevertheless, it covers a lot of specific uses: identification of single phase materials, determination of the crystal structure, identification and structural analysis of samples, recognition of amorphous materials in partially crystalline mixtures, determination of crystallite size from analysis of peak broadening, determination of crystallite shape from the study of peak symmetry and the study of thermal expansion in crystal structures using in situ heating stage equipment (Connolly 2007). Mulik et al. (2010) showed that the diffraction pattern of curcumin is significantly different from the diffraction pattern of solid lipid nanoparticles loaded with curcumin. The diffraction pattern of loaded solid lipid nanoparticles indicates that curcumin is entrapped in the lipid core of the nanoparticles, while the diffraction pattern of the unloaded solid lipid nanoparticle indicates that the addition of curcumin has not changed the nature of the solid lipid nanoparticles.

\section{Small-Angle X-ray Scattering}

Small-angle X-ray scattering (SAXS) is a technique for the study of structural features of colloidal size particles where the elastic scattering of X-rays by a sample that has inhomogeneities in the nanometric range, is recorded at very low angles (typically $0.1-10^{\circ}$ ). This angular range contains information about the shape and size of macromolecules, characteristic distances of partially ordered materials, pore sizes, etc. SAXS is capable of delivering structural information on macromolecules between 5 and 
$25 \mathrm{~nm}$ and on repeating distances in partially ordered systems of up to $150 \mathrm{~nm}$ (Glatter and Kratky 1982). Through the scattering patterns, information on the structure, shape, and size of macromolecules can be achieved. This method is non-destructive and requires a minimum sample preparation. One of the disadvantages of SAXS data analysis is that only a one-dimension scattering pattern can be obtained (Luykx et al. 2008). Jenning et al. studied the influence of the oily constituent of their particles on the subcell parameters and long spacings of solid Compritol nanocyrstals through SAXS, which was used to confirm the polymorphism behavior found through DSC measurements (Jenning et al. 2000a). Also, through SAXS, Venturini et al. showed that sorbitan monostearate is interacting with the oily phase, in the core of the solid-lipid nanoparticle (Venturini et al. 2011).

\section{Imaging Techniques}

Microscopy can be used as a direct imaging technique for nanoemulsions; nevertheless the type of microscopy used depends on the kind of matrix to be analyzed. This technique enables information regarding the size, shape, and aggregation state of the nanoemulsions. Some of the imaging methods that are used for the characterization of nanoemulsions systems are presented below.

\section{Transmission Electron Microscopy}

TEM is a technique capable of a resolution on the order of the $0.2 \mathrm{~nm}$ (Luykx et al. 2008; Wang 2000). It is widely used in the study of materials for science/metallurgy and biological sciences; in both cases the samples must be very thin and able to withstand the high vacuum present inside the instrument. Nevertheless, this technique has some drawbacks (Luykx et al. 2008; Wang 2000). Some materials require extensive sample preparation to produce a sample thin enough to be electron transparent, which makes TEM analysis a relatively time-consuming process with a low throughput of samples. The structure of the sample may be changed during the preparation process; the field of view is relatively small and the sample may be damaged by the electron beam. Bouchemal et al. studied the morphology and structure of the nanoemulsions using TEM; the combination of bright field imaging at increasing magnification and of diffraction modes were used to reveal the form and size of the emulsions and to determine the amorphous or crystalline character of the components. They direct observation enabled the possibility to perform selected area electron diffraction in order to check the crystallinity of the emulsion core components (Bouchemal et al. 2004). Chu et al. (2007) observed the microstructure and the particle-size distribution in their nanoemulsions, concluding that $\beta$-carotene particles exhibited spherical morphology with a mean diameter of $20 \mathrm{~nm}$, confirming the results obtained by DLS.

\section{Scanning Electron Microscopy}

SEM is capable of producing high-resolution images of a sample surface (Luykx et al. 2008; Reimer 2000). SEM images have a characteristic three-dimensional appearance and are useful for judging the surface structure. Generally, TEM resolution is about an order of magnitude higher than SEM resolution; however, because the SEM image relies on surface processes rather than transmission, it is able to image bulky samples and has a much greater depth of field and so can produce images that are a good representation of the $3 \mathrm{D}$ structure of the sample. In particular, the significantly large depth of field available in SEM allows a large amount of the sample to be in focus at one time. It is also capable to produce high-resolution images at higher magnification. The combination of higher magnification, larger depth of field, greater resolution, and ease of sample observation makes SEM one of the most heavily used methods in research areas today (Luykx et al. 2008). Nevertheless, SEM is also an expensive technique and requires high vacuum and a relatively high sample conductivity (Luykx et al. 2008). The presence of surfactants during nanoemulsions preparation can sometimes inhibit their characterization via SEM due to the formation of a smooth camouflaging coating on the particle surfaces (Luykx et al. 2008). Tan and Nakajima (2005b) observed great differences between coarse and nanodispersed $\beta$ carotene samples through SEM. The analysis of coarse crystalline $\beta$-carotene showed particles with irregular shapes and sizes ranging from a few $\mu \mathrm{m}$ to more than $10 \mu \mathrm{m}$, as opposed to the nanodispersions. SEM particle sizes were confirmed through DLS.

\section{Atomic Force Microscopy}

AFM is a more recently developed microscopy technique (Edwards and Baeumner 2006; Luykx et al. 2008; Ruozi et al. 2005). The high resolution $( \pm 0.1 \mathrm{~nm})$ achieved by AFM has been used to directly view single atoms or molecules that have dimensions of a few nanometers. AFM relies on the raster scanning of a nanometer-sized sharp probe over a sample that has been immobilized onto a carefully selected surface (mica or glass), resulting in a high-resolution threedimensional profile of the surface under study. AFM has the advantage of imaging almost any type of surface, including polymers, ceramics, composites, glass, and biological samples. AFM allows biomolecules to be imaged not only under physiological conditions but also during biological processes. The advantage of directly observing 
biomolecular systems in their native environment opens the possibility of analyzing their structural and functional properties at the submolecular level. Surface irregularities observed by SEM are absent in AFM inspection. AFM's disadvantages are related with the analysis of surfaces which are soft, sticky, or have loose particles floating, as the tip is in direct contact with the actual surface and will run into difficulties in those cases. Nevertheless, AFM works on most materials and has been used in a range of applications from biology and chemistry, to electronics. AFM images are complementary to other established techniques. AFM can be used for the structural characterization of, e.g., proteins, polysaccharides, and liposomes (Luykx et al. 2008; Moraru et al. 2003). Preetz et al. (2010) showed the differences between nanoemulsions and nanocapsules by studying the shape, morphology and mechanical properties of the emulsion and capsule shell through AFM, and was able to demonstrate that the shell around an oil droplet solidified with increasing amounts of polyelectrolytes.

\section{Nanoemulsions: Industrial Perspectives and Applications in the Food Market}

In recent years, nanotechnology has developed into a largescale business, becoming a multimillionaire industry. It is expected that the overall impact of the nanotechnology market will reach one trillion U.S. dollars by 2015 , housing about 2 million workers (Neethirajan and Jayas 2011; Roco and Bainbridge 2001). It is also evident through a number of reports, analysis and patent applications, that nanotechnology already has a large impact on different aspects of food and associated industries (Chen et al. 2006; Neethirajan and Jayas 2011). A report suggested that the number of companies that currently make use of nanotechnology in the food sector may be about 400 (Chau et al. 2007; Neethirajan and Jayas 2011).

Nanoemulsion production for encapsulation and delivery of functional compounds is one of the emerging fields of nanotechnology applied to food industry. Application examples are given below.

NutraLease, a technology start-up company established by a scientific team, is working to improve the bioavailability of functional compounds. Beverages containing encapsulated functional compounds such as coenzyme Q10, lycopene, lutein, $\beta$-carotene, omega-3, vitamins A, D3 and E, phytosterols and isoflavones are available (NutraLease 2011a). Their technology is based on selfassembled nanoemulsions where a better encapsulation rate as well as an improved bioavailability in the human body can be achieved (Halliday 2007; NutraLease 2011b). NutraLease nanoemulsions can protect flavor compounds from manufacturing conditions and throughout the beverages' shelf-life. It is claimed that nanoemulsions can capture the flavor and protect it from temperature, oxidation, enzymatic reactions and hydrolysis and are thermodynamically stable at a wide range of $\mathrm{pH}$ values (NutraLease 2011c).

Another example of nanotechnology in the food industry is provided by Aquanova, with their NovaSol beverage solutions (AquaNova 2011; Halliday 2007). NovaSol portfolio is divided into two categories: healthy functional compounds (coenzyme Q10, DL- $\alpha$-tocopherol acetate, vitamins $\mathrm{A}, \mathrm{D}, \mathrm{D} 3, \mathrm{E}$, and $\mathrm{K}$ and omega three fatty acids) and natural colorants ( $\beta$-carotene, apocarotenal, chlorophyll, curcumin, lutein, and sweet pepper extract) (AquaNova 2011). Aquanova claims enhanced stability (both in terms of $\mathrm{pH}$ and temperature) of encapsulated functional compounds and standardized additive concentrations (AquaNova 2011).

Some of the giants of the food industry such as Unilever and Nestlé are also applying nanotechnology to their food products. Unilever has made ice cream healthier without compromising on taste through the application of nanoemulsions. The objective is to produce ice cream with lower fat content, achieving a fat reduction from the actual $16 \%$ to $1 \%$ (Martins et al. 2007; Unilever 2011). Nestlé has a patent in water-in-oil emulsions $(10-500 \mathrm{~nm})$, aiming at achieving quicker and simpler thawing through the addition of polysorbates and other micelle-forming substances; these are claimed to contribute to an uniform thawing of frozen foods in the microwave (Möller et al. 2009).

Other applications of nanoemulsions into the food industry include antimicrobial nanoemulsions for decontamination of food equipment, packaging or food (Center for Biological Nanotechnology 2001; Gruère et al. 2011). Also being evaluated is the possibility of delivering hydrophilic or hydrophobic compounds (functional compounds), in order to improve their solubility and bioavailability (Robinson and Morrison 2009).

Despite of nanotechnology already being applied to the food industry, there is still a major gap in the regulatory framework, and most countries are still relying on existing legislation to regulate nanomaterials (Gruère et al. 2011). Improving the actual legislation framework is a crucial step to prevent consumers' misinformation regarding nanotechnology applied to foods. Such misinformation was the main responsible for the actual suspicions that many consumers have towards genetically modified organisms and their use in foods; a great deal of care is needed to prevent that from happening with nanotechnology. While legislation is still being adapted, other actions may be taken to improve consumers' confidence in foods integrating nanotechnologybased ingredients, such as the guarantee of use of food-grade materials for nanosystems production.

Another important aspect is the cost: scaling-up of highenergy approaches is still very expensive due the pressures needed to process high volumes. Such costs need to be 
reduced, either by further technical improvements in the methods or by adoption of simpler methods of nanoemulsion production. As a general recommendation, when applied to the food industry nanotechnology needs to be affordable, simple to use and with readily perceived advantages in order to be a real alternative to the standard methodologies.

\section{Conclusion}

Nanoemulsions constitute one of the most promising systems to improve solubility, bioavailability, and functionality of hydrophobic compounds. Food industry seeks to use these systems for the incorporation of, e.g., lipophilic functional compounds in food matrices.

While there are various techniques available to produce and characterize nanoemulsions, some of them have shown to be more suitable than others. In short, HPLC can be used for quantification of functional compounds, DLS may quickly determine the hydrodynamic diameter of nanoparticles in a nanoemulsion, zeta potential can indicate the stability of the nanoemulsions and TEM may be used to confirm the hydrodynamic diameter given by DLS technique and to have a general image of the nanoemulsion structure.

The application of nanoemulsions to food systems still poses challenges that need to be addressed both in terms of the production process, especially their cost, and of the characterization of both the resulting nanoemulsions and the food systems to which they will be applied in terms of product safety and acceptance.

Acknowledgment M.A. Cerqueira (SFRH/BPD/72753/2010) is recipient of a fellowship from the Fundação para a Ciência e Tecnologia (FCT, Portugal).

\section{References}

Acosta, E. (2009). Bioavailability of nanoparticles in nutrient and nutraceutical delivery. Current Opinion in Colloid \& Interface Science, 14(1), 3-15.

Akoh, C. C., \& Min, D. B. (2002). Food lipids: Chemistry, nutrition, and biotechnology (2nd ed.). New York: Marcel Dekker.

Anton, N., Gayet, P., Benoit, J.-P., \& Saulnier, P. (2007). Nano-emulsions and nanocapsules by the PIT method: An investigation on the role of the temperature cycling on the emulsion phase inversion. International Journal of Pharmaceutics, 344(1-2), 44-52.

Anton, N., Benoit, J.-P., \& Saulnier, P. (2008). Design and production of nanoparticles formulated from nano-emulsion templates-A review. Journal of Controlled Release, 128(3), 185-199.

AquaNova (2011). Available at: http://www.aquanova.de/media/public/ pdf_produkte unkosher/NovaSOL_beverage.pdf. Accessed 13 April 2011.

Araújo, F. A., Kelmann, R. G., Araújo, B. V., Finatto, R. B., Teixeira, H. F., \& Koester, L. S. (2011). Development and characterization of parenteral nanoemulsions containing thalidomide. European Journal of Pharmaceutical Sciences, 42(3), 238-245.
ASTM (1985) Zeta potential of colloids in water and waste water. American Society for Testing and Materials, D 4187-4182.

Azároff, L. V., Kaplow, R., Kato, N., Weiss, R. J., Wilson, A. J. C., \& Young, R. A. (1974). Crystal physics, diffraction, theoretical and general crystallography. New York: McGraw-Hill.

Bouchemal, K., Briançon, S., Perrier, E., \& Fessi, H. (2004). Nanoemulsion formulation using spontaneous emulsification: Solvent, oil and surfactant optimisation. International Journal of Pharmaceutics, 280(1-2), 241-251.

Burapapadh, K., Kumpugdee-Vollrath, M., Chantasart, D., \& Sriamornsak, P. (2010). Fabrication of pectin-based nanoemulsions loaded with itraconazole for pharmaceutical application. Carbohydrate Polymers, 82(2), 384-393.

Casadei, M. A., Cerreto, F., Cesa, S., Giannuzzo, M., Feeney, M., Marianecci, C., et al. (2006). Solid lipid nanoparticles incorporated in dextran hydrogels: A new drug delivery system for oral formulations. International Journal of Pharmaceutics, 325(1-2), 140-146.

Center for Biological Nanotechnology (2001). Available at: http:// www.vitamincity.com/umichnanobio.htm. Accessed 12 April 2011.

Chaix, C., Pacard, E., Elaïssari, A., Hilaire, J.-F., \& Pichot, C. (2003). Surface functionalization of oil-in-water nanoemulsion with a reactive copolymer: Colloidal characterization and peptide immobilization. Colloids and Surfaces. B, Biointerfaces, 29(1), $39-52$.

Chau, C.-F., Wu, S.-H., \& Yen, G.-C. (2007). The development of regulations for food nanotechnology. Trends in Food Science \& Technology, 18(5), 269-280.

Chen, L., Remondetto, G. E., \& Subirade, M. (2006). Food proteinbased materials as nutraceutical delivery systems. Trends in Food Science \& Technology, 17(5), 272-283.

Cheong, J. N., Tan, C. P., Man, Y. B. C., \& Misran, M. (2008). [alpha]-Tocopherol nanodispersions: Preparation, characterization and stability evaluation. Journal of Food Engineering, 89 (2), 204-209.

Chu, B.-S., Ichikawa, S., Kanafusa, S., \& Nakajima, M. (2007). Preparation of protein-stabilized $\beta$-carotene nanodispersions by emulsification-evaporation method. Journal of the American Oil Chemists' Society, 84(11), 1053-1062.

Connolly JR (2007). Introduction to X-Ray Powder Diffraction., Available at: http://epswww.unm.edu/xrd/xrdclass/01-XRD-Intro. pdf. Accessed 14 January 2011.

Date, A. A., Desai, N., Dixit, R., \& Nagarsenker, M. (2010). Selfnanoemulsifying drug delivery systems: Formulation insights, applications and advances. Nanomedicine, 5(10), 1595-1616.

de Araújo, S. C., de Mattos, A. C. A., Teixeira, H. F., Coelho, P. M. Z., Nelson, D. L., \& de Oliveira, M. C. (2007). Improvement of in vitro efficacy of a novel schistosomicidal drug by incorporation into nanoemulsions. International Journal of Pharmaceutics, $337(1-2), 307-315$.

Dulog, L., \& Schauer, T. (1996). Field flow fractionation for particle size determination. Progress in Organic Coatings, 28(1), 25-31.

Edwards, K. A., \& Baeumner, A. J. (2006). Analysis of liposomes. Talanta, 68(5), 1432-1441.

Ee, S. L., Duan, X., Liew, J., \& Nguyen, Q. D. (2008). Droplet size and stability of nano-emulsions produced by the temperature phase inversion method. Chemical Engineering Journal, 140(1-3), 626-631.

Fasolo, D., Schwingel, L., Holzschuh, M., Bassani, V., \& Teixeira, H. (2007). Validation of an isocratic LC method for determination of quercetin and methylquercetin in topical nanoemulsions. Journal of Pharmaceutical and Biomedical Analysis, 44(5), 1174-1177.

Freitas, S., Merkle, H. P., \& Gander, B. (2005). Microencapsulation by solvent extraction/evaporation: Reviewing the state of the art of microsphere preparation process technology. Journal of Controlled Release, 102(2), 313-332. 
Gao, F., Zhang, Z., Bu, H., Huang, Y., Gao, Z., Shen, J., et al. (2011). Nanoemulsion improves the oral absorption of candesartan cilexetil in rats: Performance and mechanism. Journal of Controlled Release, 149(2), 168-174.

Garti, N., Spernath, A., Aserin, A., \& Lutz, R. (2005). Nano-sized selfassemblies of nonionic surfactants as solubilization reservoirs and microreactors for food systems. Soft Matter, 1(3), 206-218.

Glatter O \& Kratky O, (1982). Small Angle X-ray Scattering. Academic Press.

Grigoriev, D. O., \& Miller, R. (2009). Mono- and multilayer covered drops as carriers. Current Opinion in Colloid \& Interface Science, 14(1), 48-59.

Gruère G, Narrod C \& Abbott L (2011). Agricultural, Food, and Water Nanotechnologies for the Poor, Available at: http://www.ifpri.org/ sites/default/files/publications/ifpridp01064.pdf. Accessed

Gutiérrez, J. M., González, C., Maestro, A., Solè, I., Pey, C. M., \& Nolla, J. (2008). Nano-emulsions: New applications and optimization of their preparation. Current Opinion in Colloid \& Interface Science, 13(4), 245-251.

Halliday J (2007). EFSA opens the floor on nanotechnology, Available at: http://www.foodnavigator.com/Financial-Industry/ EFSA-opens-the-floor-on-nanotechnology. Accessed 13 April 2011

Horn, D., \& Rieger, J. (2001). Organic nanoparticles in the aqueous phase-theory, experiment, and use. Angewandte Chemie, International Edition, 40(23), 4330-4361.

Howe, A. M., \& Pitt, A. R. (2008). Rheology and stability of oil-inwater nanoemulsions stabilised by anionic surfactant and gelatin 2 ) addition of homologous series of sugar-based co-surfactants. Advances in Colloid and Interface Science, 144(1-2), 30-37.

Huang, Q., Yu, H., \& Ru, Q. (2010). Bioavailability and delivery of nutraceuticals using nanotechnology. Journal of Food Science, 75(1), R50-R57.

Izquierdo, P., Esquena, J., Tadros, T. F., Dederen, C., Garcia, M. J., Azemar, N., et al. (2001). Formation and stability of nanoemulsions prepared using the phase inversion temperature method. Langmuir, 18(1), 26-30.

Izquierdo, P., Esquena, J., Tadros, T. F., Dederen, J. C., Feng, J., Garcia-Celma, M. J., et al. (2004). Phase behavior and nanoemulsion formation by the phase inversion temperature method. Langmuir, 20(16), 6594-6598.

Izquierdo, P., Feng, J., Esquena, J., Tadros, T. F., Dederen, J. C., Garcia, M. J., et al. (2005). The influence of surfactant mixing ratio on nano-emulsion formation by the pit method. Journal of Colloid and Interface Science, 285(1), 388-394.

Jafari, S. M., He, Y., \& Bhandari, B. (2007). Production of sub-micron emulsions by ultrasound and microfluidization techniques. Journal of Food Engineering, 82(4), 478-488.

Jenning, V., Mäder, K., \& Gohla, S. H. (2000). Solid lipid nanoparticles (SLN(TM)) based on binary mixtures of liquid and solid lipids: a 1H-NMR study. International Journal of Pharmaceutics, 205(1-2), 15-21.

Jenning, V., Thünemann, A. F., \& Gohla, S. H. (2000). Characterisation of a novel solid lipid nanoparticle carrier system based on binary mixtures of liquid and solid lipids. International Journal of Pharmaceutics, 199(2), 167-177.

Jiahui, H., Johnston, K. P., \& Williams Iii, R. O. (2004). Nanoparticle engineering processes for enhancing the dissolution rates of poorly water soluble drugs. Drug Development and Industrial Pharmacy, 30(3), 233-245.

Jores, K., Mehnert, W., Drechsler, M., Bunjes, H., Johann, C., \& Mäder, K. (2004). Investigations on the structure of solid lipid nanoparticles (SLN) and oil-loaded solid lipid nanoparticles by photon correlation spectroscopy, field-flow fractionation and transmission electron microscopy. Journal of Controlled Release, 95(2), 217-227.
Katagi, S., Kimura, Y., \& Adachi, S. (2007). Continuous preparation of $\mathrm{O} / \mathrm{W}$ nano-emulsion by the treatment of a coarse emulsion under subcritical water conditions. LWT - Food Science and Technology, 40(8), 1376-1380.

Kelmann, R. G., Kuminek, G., Teixeira, H. F., \& Koester, L. S. (2007). Carbamazepine parenteral nanoemulsions prepared by spontaneous emulsification process. International Journal of Pharmaceutics, 342(1-2), 231-239.

Kesisoglou, F., Panmai, S., \& Wu, Y. H. (2007). Application of nanoparticles in oral delivery of immediate release formulations. Current Nanoscience, 3, 183-190.

Lee, S. J., \& McClements, D. J. (2010). Fabrication of proteinstabilized nanoemulsions using a combined homogenization and amphiphilic solvent dissolution/evaporation approach. Food Hydrocolloids, 24(6-7), 560-569.

Leong, T. S. H., Wooster, T. J., Kentish, S. E., \& Ashokkumar, M. (2009). Minimising oil droplet size using ultrasonic emulsification. Ultrasonics Sonochemistry, 16(6), 721-727.

Liu, W., Sun, D., Li, C., Liu, Q., \& Xu, J. (2006). Formation and stability of paraffin oil-in-water nano-emulsions prepared by the emulsion inversion point method. Journal of Colloid and Interface Science, 303(2), 557-563.

Luykx, D. M. A. M., Goerdayal, S. S., Dingemanse, P. J., Jiskoot, W., \& Jongen, P. M. J. M. (2005). HPLC and tandem detection to monitor conformational properties of biopharmaceuticals. Journal of Chromatography B, 821(1), 45-52.

Luykx, D. M. A. M., Peters, R. J. B., van Ruth, S. M., \& Bouwmeester, H. (2008). A review of analytical methods for the identification and characterization of nano delivery systems in food. Journal of Agricultural and Food Chemistry, 56(18), 8231-8247.

Maa, Y.-F., \& Hsu, C. C. (1999). Performance of sonication and microfluidization for liquid-liquid emulsification. Pharmaceutical Development and Technology, 4(2), 233-240.

Martins P, Dulley R, Ramos S, Barbosa M, Assumpção R, Junior S \& Lacerda A (2007). Nanotecnologias na indústria de alimentos., Available at: http://www.pucsp.br/eitt/downloads/vi_ciclo_paulomartins_marisabarbosa_nano_puc.pdf. Accessed 14 April 2011.

McClements, D. J. (2000). Isothermal titration calorimetry study of pectin-ionic surfactant interactions. Journal of Agricultural and Food Chemistry, 48(11), 5604-5611.

McClements, D. (2005). Food emulsions: Principles, practice, and techniques (2nd ed.). Boca Raton: CRC.

McClements, D. J., Decker, E. A., \& Weiss, J. (2007). Emulsion-based delivery systems for lipophilic bioactive components. Journal of Food Science, 72(8), R109-R124.

McClements, D. J., Decker, E. A., Park, Y., \& Weiss, J. (2009). Structural design principles for delivery of bioactive components in nutraceuticals and functional foods. Critical Reviews in Food Science and Nutrition, 49(6), 577-606.

Mills, I., Cvitas, T., Homann, K., Kallay, N., \& Kuchitsu, K. (1993). IUPAC quantities, units and symbols in physical chemistry (2nd ed.). Oxford: Blackwell.

Möller, M., Eberle, U., Hermann, A., Moch, K., \& Stratmann, B. (2009). Nanotechnology in the food sector. Zürich: TA-SWISS.

Morales, D., Gutiérrez, J. M., García-Celma, M. J., \& Solans, Y. C. (2003). A study of the relation between bicontinuous microemulsions and oil/water nano-emulsion formation. Langmuir, 19 (18), 7196-7200.

Moraru, C. I., Panchapakesan, C. P., Huang, Q., Takhistov, P., Liu, S., \& Kokini, J. L. (2003). Nanotechnology, a new frontier in food science. Food Technology, 57(12), 24-29.

Mulik, R. S., Mönkkönen, J., Juvonen, R. O., Mahadik, K. R., \& Paradkar, A. R. (2010). Transferrin mediated solid lipid nanoparticles containing curcumin: Enhanced in vitro anticancer activity by induction of apoptosis. International Journal of Pharmaceutics, 398(1-2), 190-203. 
Neethirajan, S., \& Jayas, D. (2011). Nanotechnology for the food and bioprocessing industries. Food and Bioprocess Technology, 4(1), $39-47$.

Nicolet T (2001). Introduction to fourier transform infrared spectrometry., Available at: http://mmrc.caltech.edu/FTIR/FTIRintro.pdf. Accessed 11 January 2011.

NutraLease (2011a). Available at: http://www.nutralease.com/Nutra/ Templates/showpage. asp?DBID $=1 \&$ LNGID $=1 \&$ TMID $=84 \&$ FID=767. Accessed 13 April 2011.

NutraLease (2011b). Available at: http://www.nutralease.com/Nutra/ Templates/showpage.asp?DBID $=1 \&$ LNGID $=1 \&$ TMID $=84 \&$ FID=769. Accessed 13 April 2011.

NutraLease (2011c). Available at: http://www.nutralease.com/Nutra/ Templates/showpage. asp?DBID $=1 \&$ LNGID $=1 \&$ TMID $=84 \&$ FID $=768$. Accessed 13 April 2011.

Pan, X., Yao, P., \& Jiang, M. (2007). Simultaneous nanoparticle formation and encapsulation driven by hydrophobic interaction of casein-graft-dextran and [beta]-carotene. Journal of Colloid and Interface Science, 315(2), 456-463.

Porras, M., Solans, C., González, C., \& Gutiérrez, J. M. (2008). Properties of water-in-oil (W/O) nano-emulsions prepared by a low-energy emulsification method. Colloids and Surfaces A: Physicochemical and Engineering Aspects, 324(1-3), 181-188.

Preetz, C., Hauser, A., Hause, G., Kramer, A., \& Mäder, K. (2010). Application of atomic force microscopy and ultrasonic resonator technology on nanoscale: Distinction of nanoemulsions from nanocapsules. European Journal of Pharmaceutical Sciences, 39 (1-3), 141-151.

Quintanilla-Carvajal, M., Camacho-Díaz, B., Meraz-Torres, L., Chanona-Pérez, J., Alamilla-Beltrán, L., Jimenéz-Aparicio, A., et al. (2010). Nanoencapsulation: A new trend in food engineering processing. Food Engineering Reviews, 2(1), 39-50.

Rao, J., \& McClements, D. J. (2010). Stabilization of phase inversion temperature nanoemulsions by surfactant displacement. Journal of Agricultural and Food Chemistry, 58(11), 7059-7066.

Reimer, L. (2000). Scanning electron microscopy: physics of image formation and microanalysis, second edition. Measurement Science and Technology, 11(12), 1826.

Relkin, P., Yung, J.-M., Kalnin, D., \& Ollivon, M. (2008). Structural behaviour of lipid droplets in protein-stabilized nano-emulsions and stability of $\alpha$-tocopherol. Food Biophysics, 3(2), 163-168.

Ribeiro, H. S., Chu, B.-S., Ichikawa, S., \& Nakajima, M. (2008). Preparation of nanodispersions containing [beta]-carotene by solvent displacement method. Food Hydrocolloids, 22(1), 12-17.

Robinson D \& Morrison M (2009). Report on nanotechnology in agrifood, Available at: http://www.observatorynano.eu/project/ filesystem/files/FullReportNanotechnologyinAgrifoodMay2009. pdf. Accessed 13 April 2011.

Roco, M. C., \& Bainbridge, W. S. (2001). Societal Implications of nanoscience nanotechnology (pp. 3-4). Boston: Kluwer.

Rouessac, F., \& Rouessac, A. (2007). Chemical analysis: Modern instrumentation methods and techniques ( 2 nd ed.). France: Wiley.

Ruozi, B., Tosi, G., Forni, F., Fresta, M., \& Vandelli, M. A. (2005). Atomic force microscopy and photon correlation spectroscopy: Two techniques for rapid characterization of liposomes. European Journal of Pharmaceutical Sciences, 25(1), 81-89.

Sadtler, V., Rondon-Gonzalez, M., Acrement, A., Choplin, L., \& Marie, E. (2010). PEO-covered nanoparticles by emulsion inversion point (eip) method. Macromolecular Rapid Communications, 31(11), 998-1002.

Sadurní, N., Solans, C., Azemar, N., \& García-Celma, M. J. (2005). Studies on the formation of $\mathrm{O} / \mathrm{W}$ nano-emulsions, by low-energy emulsification methods, suitable for pharmaceutical applications. European Journal of Pharmaceutical Sciences, 26(5), 438-445.

Salmah, H., Ismail, H., \& Bakar, A. A. (2008). The effects of dynamic vulcanization and compatibilizer on properties of paper sludge- filled polypropylene/ethylene propylene diene terpolymer composites. Journal of Applied Polymer Science, 107(4), 2266-2273.

Sanguansri, P., \& Augustin, M. A. (2006). Nanoscale materials development - a food industry perspective. Trends in Food Science \& Technology, 17(10), 547-556.

Shefer, A., \& Shefer, S. (2003). Novel encapsulation system provides controlled release of ingredients. Food Technology, 57(11).

Shinoda, K., \& Saito, H. (1968). The effect of temperature on the phase equilibria and the types of dispersions of the ternary system composed of water, cyclohexane, and nonionic surfactant. Journal of Colloid and Interface Science, 26(1), 70-74.

Shinoda, K., \& Saito, H. (1969). The stability of o/w type emulsions as a function of temperature and the hlb of emulsifiers: the emulsification by pit-method. Journal of Colloid and Interface Science, 30(1), 258-263.

Silva, H. D., Cerqueira, M. A., Souza, B. W. S., Ribeiro, C., Avides, M. C., Quintas, M. A. C., et al. (2011). Nanoemulsions of [beta]carotene using a high-energy emulsification-evaporation technique. Journal of Food Engineering, 102(2), 130-135.

Simunkova, H., Pessenda-Garcia, P., Wosik, J., Angerer, P., Kronberger, H., \& Nauer, G. E. (2009). The fundamentals of nano- and submicro-scaled ceramic particles incorporation into electrodeposited nickel layers: Zeta potential measurements. Surface and Coatings Technology, 203(13), 1806-1814.

Spernath, A., \& Aserin, A. (2006). Microemulsions as carriers for drugs and nutraceuticals. Advances in Colloid and Interface Science, 128-130, 47-64.

Tadros, T., Izquierdo, P., Esquena, J., \& Solans, C. (2004). Formation and stability of nano-emulsions. Advances in Colloid and Interface Science, 108-109, 303-318.

Tan, C. P., \& Nakajima, M. (2005a). [beta]-Carotene nanodispersions: preparation, characterization and stability evaluation. Food Chemistry, 92(4), 661-671.

Tan, C. P., \& Nakajima, M. (2005b). Effect of polyglycerol esters of fatty acids on physicochemical properties and stability of $\beta$-carotene nanodispersions prepared by emulsification/evaporation method. Journal of the Science of Food and Agriculture, 85(1), 121-126.

Thanasukarn, P., Pongsawatmanit, R., \& McClements, D. J. (2004). Influence of emulsifier type on freeze-thaw stability of hydrogenated palm oil-in-water emulsions. Food Hydrocolloids, 18(6), 1033-1043.

The Commission Of The Eureopean Communities (1995). Commission Directive 95/45/EC, Available at: http://ec.europa.eu/food/fs/ sfp/addit flavor/flav13 en.pdf. Accessed 19 May 2011.

The good scents company Available at: http://www.thegoodscents company.com/data/rw1287041.html. Accessed 18 May 2011.

Ubbink, J., \& Krüger, J. (2006). Physical approaches for the delivery of active ingredients in foods. Trends in Food Science \& Technology, 17(5), 244-254.

Unilever (2011). Available at: http://www.unilever.com/innovation/ productinnovations/coolicecreaminnovations/?WT.LHNAV= Cool_ice_cream_innovations. Accessed 13 April 2011.

Usón, N., Garcia, M. J., \& Solans, C. (2004). Formation of water-inoil (W/O) nano-emulsions in a water/mixed non-ionic surfactant/ oil systems prepared by a low-energy emulsification method. Colloids and Surfaces A: Physicochemical and Engineering Aspects, 250(1-3), 415-421.

Velikov, K. P., \& Pelan, E. (2008). Colloidal delivery systems for micronutrients and nutraceuticals. Soft Matter, 4(10), 1964-1980.

Velinova, M. J., Staffhorst, R. W. H. M., Mulder, W. J. M., Dries, A. S., Jansen, B. A. J., de Kruijff, B., et al. (2004). Preparation and stability of lipid-coated nanocapsules of cisplatin: Anionic phospholipid specificity. Biochimica et Biophysica Acta (BBA) Biomembranes, 1663(1-2), 135-142.

Venturini, C. G., Jäger, E., Oliveira, C. P., Bernardi, A., Battastini, A. M. O., Guterres, S. S., et al. (2011). Formulation of lipid core 
nanocapsules. Colloids and Surfaces A: Physicochemical and Engineering Aspects, 375(1-3), 200-208.

Walstra, P. (1993). Principles of emulsion formation. Chemical Engineering Science, 48(2), 333-349.

Wang, Z. L. (2000). Transmission electron microscopy of shapecontrolled nanocrystals and their assemblies. The Journal of Physical Chemistry. B, 104(6), 1153-1175.

Weiss, J., Decker, E., McClements, D., Kristbergsson, K., Helgason, T., \& Awad, T. (2008). Solid lipid nanoparticles as delivery systems for bioactive food components. Food Biophysics, 3(2), $146-154$.

Wissing, S. A., Kayser, O., \& Müller, R. H. (2004). Solid lipid nanoparticles for parenteral drug delivery. Advanced Drug Delivery Reviews, 56(9), 1257-1272.

Wooster, T. J., Golding, M., \& Sanguansri, P. (2008). Impact of oil type on nanoemulsion formation and Ostwald ripening stability. Langmuir, 24(22), 12758-12765.
Wulff-Pérez, M., Torcello-Gómez, A., Gálvez-Ruíz, M. J., \& MartínRodríguez, A. (2009). Stability of emulsions for parenteral feeding: Preparation and characterization of $\mathrm{o} / \mathrm{w}$ nanoemulsions with natural oils and Pluronic f68 as surfactant. Food Hydrocolloids, 23(4), 1096-1102.

Yin, L.-J., Chu, B.-S., Kobayashi, I., \& Nakajima, M. (2009). Performance of selected emulsifiers and their combinations in the preparation of [beta]-carotene nanodispersions. Food Hydrocolloids, 23(6), 1617-1622.

Yuan, Y., Gao, Y., Mao, L., \& Zhao, J. (2008). Optimisation of conditions for the preparation of [beta]-carotene nanoemulsions using response surface methodology. Food Chemistry, 107(3), 1300-1306.

Yuan, Y., Gao, Y., Zhao, J., \& Mao, L. (2008). Characterization and stability evaluation of [beta]-carotene nanoemulsions prepared by high pressure homogenization under various emulsifying conditions. Food Research International, 41(1), 61-68. 